\title{
High Temperature Quantum Kinetic Effects in Silicon Nanosandwiches
}

\author{
(C) N.T. Bagraev ${ }^{1,2}$, L.E. Klyachkin ${ }^{1}$, V.S. Khromov ${ }^{1}$, A.M. Malyarenko ${ }^{1}$, V.A. Mashkov ${ }^{2}$, \\ T.V. Matveev ${ }^{2}$, V.V. Romanov ${ }^{2}$, N.I. Rul ${ }^{1,2.1}$, K.B. Taranets ${ }^{2}$ \\ ${ }^{1}$ loffe Institute, \\ 194021 St. Petersburg, Russia \\ ${ }^{2}$ Peter the Great St. Petersburg Polytechnic University, \\ 195251 St. Petersburg, Russia \\ ๑ E-mail: impurity.dipole@mail.ioffe.ru
}

The negative- $U$ impurity stripes confining the edge channels of semiconductor quantum wells are shown to allow the effective cooling inside in the process of the spin-dependent transport, with the reduction of the electron-electron interaction. The aforesaid promotes also the creation of composite bosons and fermions by the capture of single magnetic flux quanta on the edge channels under the conditions of low sheet density of carriers, thus opening new opportunities for the registration of the high temperature de Haas-van Alphen, $300 \mathrm{~K}$, quantum Hall, $77 \mathrm{~K}$, effects as well as quantum conductance staircase in the silicon sandwich structure.

\section{Acknowledgements}

The work was supported by the programme „5-100-2020“, project 6.1.1 of SPSPU (2014); project 1963 of SPbPU (2014); the programme of fundamental studies of the Presidium of the Russian Academy of Sciences „Actual problems of low temperature physics“ (grant 10.4); project 10.17 „Interatomic and molecular interactions in gases and condensed matter". 